\title{
Managing QoS for Future Internet Applications over Virtual Sensor Networks
}

\author{
Panagiotis Trakadas ${ }^{1,}$, Helen Leligou ${ }^{2}$, Theodore Zahariadis ${ }^{2}$, Panagiotis Karkazis ${ }^{3}$, \\ and Lambros Sarakis ${ }^{2}$ \\ ${ }^{1}$ ADAE, Hellenic Authority of Communication Security and Privacy, Athens, Greece \\ trakadaspeadae.gr \\ ${ }^{2}$ Dept. of Electrical Engineering, TEI of Chalkis, Psahna, Greece \\ \{zahariad, leligou, sarakis\} @teihal.gr \\ ${ }^{3}$ Dept. of Electronic \& Computer Engineering, Technical University of Crete, Greece \\ pkarkazis@isc.tuc.gr
}

\begin{abstract}
The integration of Wireless Sensor Networks (WSNs) in the Future Internet has opened new opportunities for novel applications that meet the needs of modern societies. Virtualisation of the available resources and the services offered by WSNs enables their efficient sharing between diverse applications reducing costs. Responding to this challenge, the VITRO project has designed a WSN virtualization architecture that targets to decouple the physical sensor infrastructure from the applications running on top of it. In the concept of Virtual Sensor Network platform, the WSNs are capable of collaborating among each other (even if they belong to different administrator domains or comprise of heterogeneous platforms) to flexibly support service composition and fuel novel application development. To meet the diverse Quality of Service (QoS) requirements imposed by the different applications running on top of the same infrastructure, VITRO has designed, implemented and integrated a routing solution that enables the establishment of different routing paths per application, based on different routing criteria in order to optimize the performance aspect(s) of interest to each application. In this paper, we demonstrate how the VITRO routing solution could be employed in various use cases including smart homes/buildings, smart cities, smart business environments and securityrelated applications. We evaluate the achieved performance using computer simulation results and provide guidelines for prospective users.
\end{abstract}

Keywords: wireless sensor networks, virtualization, routing, Quality of Service.

\section{Introduction}

Future Internet will interconnect trillions of devices, with a significant part being sensor nodes or nodes with sensing capabilities which cater information that enables the development of sophisticated applications towards meeting the needs of modern

* Contact information: Dr. Panagiotis Trakadas, ADAE, Hellenic Authority of Communication Security and Privacy, Athens, Greece, trakadasp@adae.gr

A. Galis and A. Gavras (Eds.): FIA 2013, LNCS 7858, pp. 52-63, 2013.

(C) The Author(s). This article is published with open access at link.springer.com 
societies and citizens. The capability of re-purposing existing Wireless Sensor Networks (WSN) - in order to cope with different tasking functionality beyond the scope of the original sensor design and deployment - and of sharing the network resources can significantly accelerate the development and execution of new applications at significantly lower cost. Responding to this challenge, the notion of Virtual Sensor Networks (VSN) has been introduced, targeting to decouple the physical sensor deployment from the applications running on top of it. In this concept, WSNs are no longer deployed to support a specific service but are capable of collaborating among them towards realizing new services and applications.

The design and development of VSNs are at the center of the VITRO project which has developed a reference architecture [1] to enable the realization of a scalable, flexible, adaptive, energy-efficient and trust-aware Virtual Sensor Network platform. In this architecture, which uses the Internet (IPv6) as the physical bearer between sensor platforms and applications, sensors are deployed by potential (public or private) organizations and can then be used to feed information to applications deployed by other organizations. However, accommodating multiple services with different requirements (e.g. QoS, security) on a shared infrastructure, without predefined resource reservations, jeopardizes the experienced QoS acceptability. For this reason, VITRO has designed a routing solution [2]-[3] that supports QoS differentiation per application and complies with the RPL protocol (Routing Protocol for Low power and lossy networks) which has recently been standardized by IETF [4], providing a formal routing algebra framework for the proper composition of routing metrics.

In this paper, we showcase how the VITRO routing solution can be used to achieve QoS differentiation for applications running over the same WSN infrastructure and how the prospective user should exploit it to optimize the performance metric of interest in different real-life application domains. The rest of the paper is organized as follows: In section 2 we briefly describe the VITRO routing solution. In section 3, we present representative application domains where sensor network virtualization is of benefit and discuss how the VITRO routing solution can be used to achieve the QoS requirements in each use case. We also provide computer simulation results to explore the flexibility of our solution and draw guidelines on the use of the proposed routing framework from prospective users. The simulation results, presented in this paper, have been validated using the integrated VITRO platform. Finally, conclusions are reached in section 4 .

\section{The VITRO Routing Solution}

To maximize exploitation potential, VITRO has decided to use standardized routing protocols as the basis for the design of a routing protocol that meets the needs of the Virtual Sensor Network concept. The so-called IPv6 Routing Protocol for Low power Lossy Networks (RPL) [4] has been standardized by the Routing Over Low power and Lossy networks (ROLL) working group of IETF. RPL provides a mechanism whereby multipoint-to-point traffic from devices inside the Low power and Lossy Network (LLN) is routed towards a central control point, as well as point-to multipoint traffic from the central control point to the devices inside the LLN. RPL 
constructs Directed Acyclic Graphs (DAG) and defines the rules based on which every node selects a neighbor as its parent in the DAG. To cover the diverse requirements imposed by different applications, ROLL has specified [5] a set of link and node routing metrics and constraints (which can be either static or dynamic), suitable to Low Power and Lossy link Networks that can be used in RPL.

For the construction of the DAG, each node calculates a Rank value and advertises it in the so-called Destination Information Object (DIO) messages. The Rank of a node is a scalar representation of the "location" of that node within a destinationoriented DAG (DODAG). In particular, the Rank of the nodes must monotonically decrease as the DODAG is followed towards the DAG destination (root). Each node selects as parent the neighbor that advertises the minimum rank value. This guarantees loop free operation and convergence. The rank has properties of its own that are not necessarily those of all metrics. It is the Objective Function (OF) that defines how routing metrics are used to compute the Rank, and as such must hold certain properties.

VITRO has proposed specific rules for quantifying five different routing metrics that capture different performance aspects and two different ways (namely the additive and lexicographic approaches) to combine them in composite routing metrics. In [2]-[3], we have also proved (using the well-established by Sobrinho et. al. routing algebra [6]) that the RPL protocol, utilizing the proposed primary and composite routing metrics, converges to optimal paths in a loop-free manner. The proposed routing metrics as well as the affected performance aspect are included in Table 1 [7].

Table 1. The VITRO primary routing metrics and the affected performance aspect

\begin{tabular}{|c|c|c|}
\hline $\begin{array}{c}\text { Primary routing } \\
\text { metric }\end{array}$ & Routing operation & Affected performance metric \\
\hline \multicolumn{2}{|c|}{ Hop Count (HC) Shortest path } & Low latency \\
\hline $\begin{array}{l}\text { Expected } \\
\text { Transmission } \\
\text { Count (ETX) }\end{array}$ & $\begin{array}{l}\text { More reliable link with respec } \\
2 \text { performance }\end{array}$ & $\begin{array}{l}\text { rLow packet loss - Low la- } \\
\text { tency }\end{array}$ \\
\hline
\end{tabular}

Received SignalMore reliable link with respect to sig-Increased reliability with Strength Indica-nal strength (does not take into accountrespect to transmissions suction (RSSI) congestion) cessfully received by neighbours, high latency

Packet Forward-Selection of the most reliableEfficient detection of misbeing Indicationneighbour for forwarding traffic pack-having nodes, low packet (PFI) ets loss due to misbehaviours

Remaining En-Avoids systematically using the sameNetwork lifetime elongation ergy (RE) neighbours for forwarding, leading to energy depletion

VITRO capitalizes on an important feature of RPL which is the support of different DODAG instances. RPL defines that different DODAG instances can co-exist over 
the same infrastructure and each instance can be formed based on different routing metrics thus enabling the optimization of different performance aspects. As shown in Figure 1, the infrastructure (shown at the bottom of the figure) consisting of eight (8) nodes is used to form two different VSNs supporting two different applications. Different routing metrics, reflected in different Objective Functions, can be used to guide the construction of each DODAG to optimize different performance aspects. As shown in Figure 1, depending on the supported OFs, each node can participate in one or two VSNs, constructing two separate but concurrent routing graphs. (The objective functions OF0 MRHOF are defined in [8] and [9] respectively.

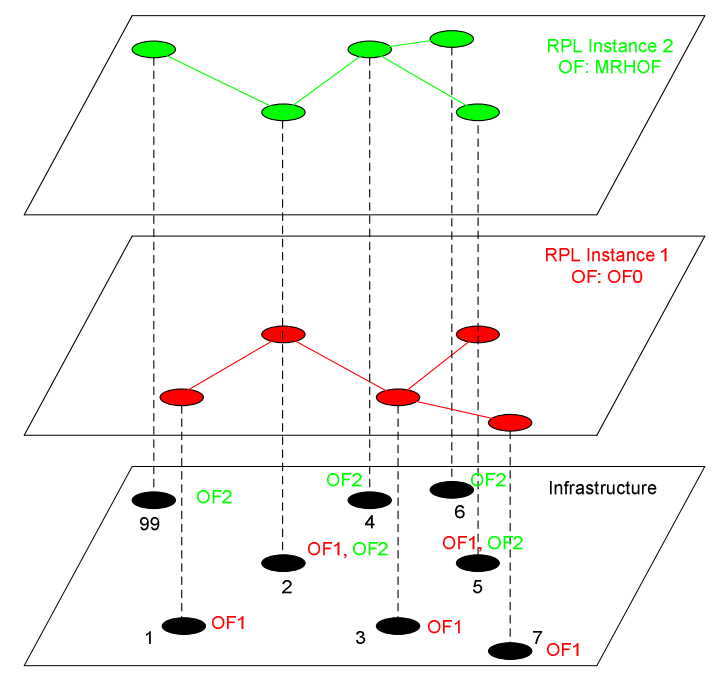

Fig. 1. Two VSN instantiations within the same WSI

\section{Applications Exploiting Virtualization of WSNs}

As virtualization targets the re-usability of sensing devices for different purposes, it is important to identify the routing metrics (primary or composite) that better match specific application domains. Our aim is to define implementable rules and thus enable effective exploitation of the potential of the VITRO routing solution for a wide set of applications. Thus, further and in-depth investigation on routing metrics composition methods and individual weights for satisfying specific QoS requirements arising from the specific application areas under examination will be presented, supported by results obtained through simulation.

The application domains selected by the VITRO consortium and used to define application and system requirements are included in Table 2. Each application requires either service virtualization, or resource virtualization or service composition or any combination of the previous. 
Table 2. Indicative application domains exploiting VSN capabilities

\begin{tabular}{|c|c|}
\hline Application Domain & Application \\
\hline \multirow{2}{*}{$\begin{array}{l}\text { Energy control in public and private } \\
\text { buildings }\end{array}$} & Energy efficient smart schools/buildings \\
\hline & Energy efficient homes \\
\hline \multirow[b]{2}{*}{ Traffic control } & Traffic light control \\
\hline & $\begin{array}{l}\text { City traffic management through Variable Message } \\
\text { Signs }\end{array}$ \\
\hline Logistics & Product monitoring in the production to sales chain \\
\hline \multirow{3}{*}{ Security } & Disaster management \\
\hline & Security in public spaces and buildings \\
\hline & $\begin{array}{l}\text { Baggage proximity checker for security and theft } \\
\text { prevention }\end{array}$ \\
\hline
\end{tabular}

\subsection{Simulation Environment}

Before proceeding with the analysis of the simulation results, it is necessary to describe the employed simulation environment. We modelled the VITRO routing solution based on the JSIM [10] open simulation platform and the features of our simulator (available in [11] as open source) are presented in [12]. The network topology consisted of 1000 nodes with one node acting as sink node. We ran several tests with different configurations and parameters in order to capture many characteristics of the network. In detail, tests have been performed for 50, 100, 200 and 500 sessions (number of nodes generating sensing data and sending them to the sink node). This provided the ability to check the network performance under low and high traffic conditions. In all tests, the sensing nodes were kept the same (mainly positioned in the perimeter of the network topology), while the rest of the nodes were randomly placed for each set of tests. Moreover, we ran different tests for several number of misbehaving (either acting maliciously or simply malfunctioning) nodes (50, 100, and 150), uniformly distributed over the network and equally split among a variety of possible misbehaving situations (reduced transmission power, security enabled, missed ACKs, not forwarding packets, etc.). The performance metrics we focused on were latency and packet loss percentage.

\subsection{Energy Control in Public and Private Buildings}

As energy consumption control is an ultimate priority of our society, applications that collect energy demand information in order to intelligently schedule the energy consumption. The sensors are deployed in buildings (e.g. schools, office buildings and homes) and usually need to detect human and device activities through sensing devices which can be of different technology, possibly initially installed for different 
reasons (e.g. security). In such situations, VITRO developments offer seamless access to all sensing entities.

For this type of applications, performance optimisation should deal with reducing latency and extending network lifetime to efficiently tackle scalability issues arising in such networks, as the number of nodes may reach thousands in large business environments. Thus, we consider that the routing metric of interest includes composite routing metrics based on either (HC, RE) or (RSSI, RE). For each pair of metrics, we have tested their lexicographical combination and their combination in a weighted sum for different weight values. The results with respect to packet latency for (HC, RE) and (RSSI, RE) combinations are shown in Figure 2.

From Figure 2 it can be observed that, in general, the lexicographic approach incurs less latency, compared to the additive approaches, since path related attributes (namely HC and RSSI) are inspected with absolute priority over RE. Comparing latency values between (HC, RE) and (RSSI, RE) combinations, a non-negligible difference is observed (almost double). This is explained as the path in (RSSI, RE) case is longer and traverses a higher number of nodes. This is because under the RSSI metric, a node prefers as a parent a node being closer (larger value of advertised transmission power) having a direct impact on the length of the traversed path measured in number of hops. Additionally, adopting RSSI as the primary routing metric implies that for the transmission of a message a total number of transmissions in the network is higher than in the case where $\mathrm{HC}$ is adopted. As a conclusion, either a lexical (HC, RE) combination or an additive (HC, RE) approach where the RE metric weighting factor will not exceed 0.4 is the optimal choice for this application domain.
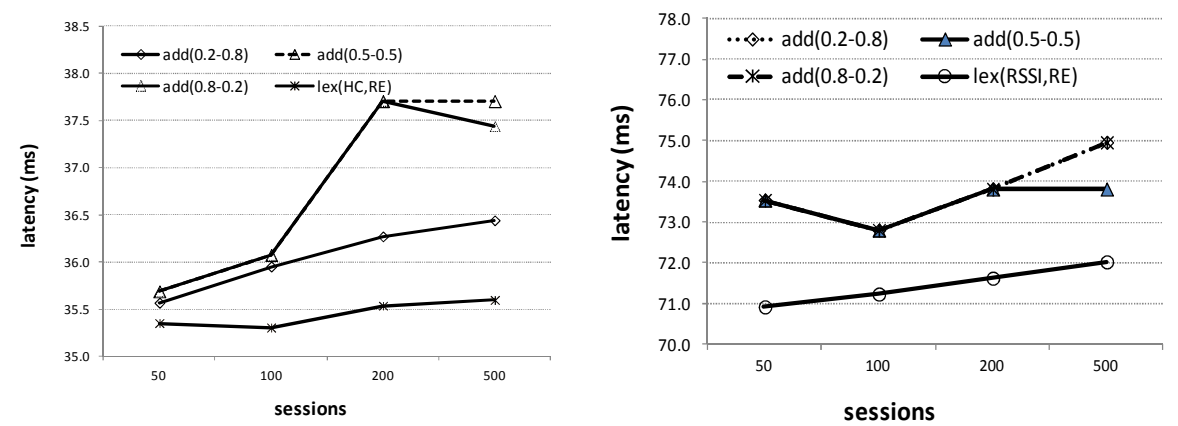

Fig. 2. Simulation results for additive and lexicographic (HC, RE) metric composition on the left and (RSSI, RE) metric composition on the right in terms of latency for 50 misbehaving nodes in the network

\subsection{Traffic Control Application Domain}

The city traffic management and the traffic light control applications can be considered as representative examples of applications using the same sensing nodes. To manage the city traffic and control the traffic lights, information such as the dynamically changing traffic conditions, the environmental conditions (e.g. temperature, humidity, $\mathrm{CO}_{2}$ emissions) has to be collected. It is obvious that this type of information is of 
interest to a great variety of stakeholders/authorities, from municipality to environment related authorities. VITRO makes possible their re-use to save budgets and space in the sidewalk.

In applications such as traffic light control and traffic management through variable message signs, the requirements are quite different from the previous case. More specifically, these applications require a high level of security along with a minimization of latency. Providing traffic control and management requires that measurements and signals must be delivered without delay and with a minimum packet loss percentage. As this is a delay-sensitive application, RSSI is not appropriate. Instead, HC and PFI (which is a security related metric defined in [3] are selected.

The results for different composite metrics of $\mathrm{HC}$ and PFI are included in Figures 3 and 4 for 50 and 150 misbehaving nodes respectively. Observing Figure 3, it is obvious that the lexicogaphic combination of PFI and HC results in significantly higher latency and packet loss than all the additive composition metrics approach, because, as it is related solely with neighbour forwarding reliability, it does not manage to guide the construction of a path to the destination. For this reason, we have excluded lex(PFI, HC) from Figure 4.

Comparing the two figures, it is evident, as expected, that the performance degrades as the number of misbehaving nodes increases in terms of both latency and packet loss. For 50 misbehaving nodes, for any number of sessions the latency remains lower than $36 \mathrm{~ms}$ while for 150 misbehaving nodes the latency reaches the values of $37,5 \mathrm{~ms}$. More importanly, the packet loss for 50 misbehaving nodes reamins well below $20 \%$ while for 150 misbehaving nodes (called misbehaving nodes), packet loss reaches $23 \%$.
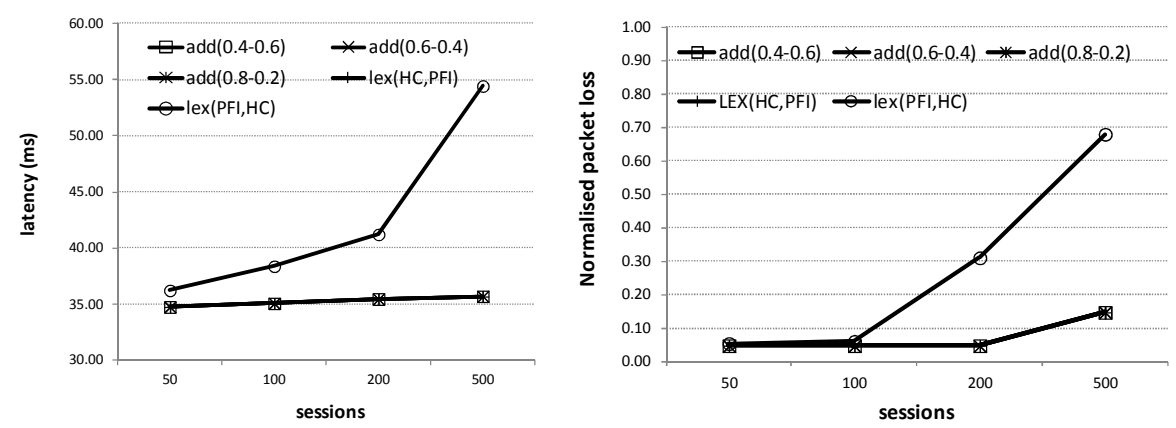

Fig. 3. Latency (left) and packet loss (right) results for additive and lexicographic (HC, PFI) approaches for varying number of sessions in the presence of 50 misbehaving nodes

Apart from the lexicographic (PFI, HC) composite routing metric, the inclusion of PFI in the objective function leads to the efficient detection of misbehaving nodes. It is worth pointing out that the existence of even one misbehaving node on the path, would result in $100 \%$ packet loss for all flows traversing this node in the absence of PFI metric. Taking into account PFI, the packet loss is kept below $25 \%$ even when 
150 misbehaving packet exist in the network and the number of active sessions reach 500 (as shown in Figure 4, right hand side).

The results reveal that any combination of $\mathrm{HC}$ and PFI in additive manner leads to stable performance and the weighting factors slightly affect the achieved performance.
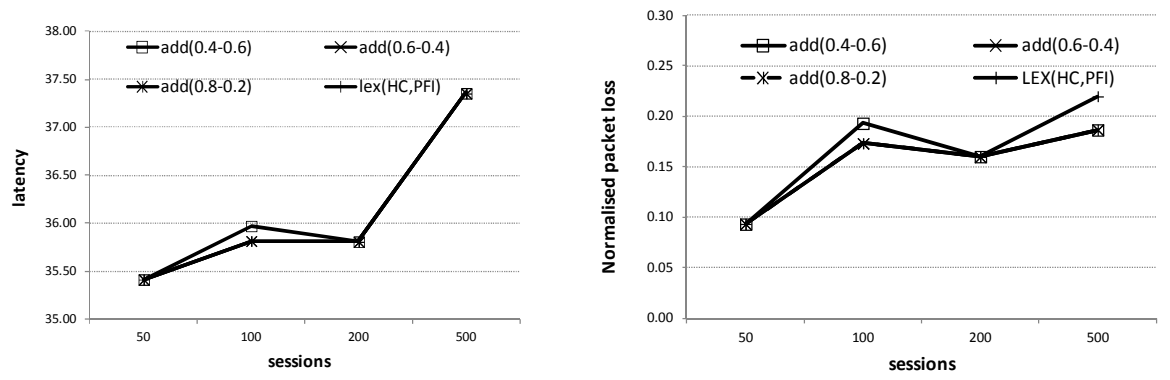

Fig. 4. Latency (left) and packet loss (right) results for additive and lexicographic (HC, PFI) approaches for a varying number of sessions in the presence of 100 misbehaving nodes

\subsection{Logistics Applications}

The domain of logistics is constantly increasing its indices in Europe with product monitoring being at the focal point of this application area. The automated monitoring of the product saves significant time and hence cost for searching products in wide and distributed even among different cities warehouses.

The applications running on top of the sensing infrastructures require high reliability in an area of dense WSNs and long system lifetime. To meet these requirements, the use of a composite routing metric combining ETX (to minimize retransmissions in this dense environment) and remaining energy (to prolong the network lifetime) is proposed.

The performance results for combinations of ETX and RE routing metrics are depicted in Figure 5. It is observed that the additive approaches perform better than lexicographic ones, for both latency and packet loss performance metrics, even for high traffic cases (high number of active sessions). The observed packet loss is attributed to the absence of a security routing metric (PFI) in the presence of misbehaving nodes. As a result, nodes suppressing data packets cannot be detected and thus, excluded from forwarding. Instead, link reliability as well as distributed energy consumption drives the routing decision. Compared to the previous use case, where link reliability was not taken into account, latency in this case is significantly decreased, as dictated by ETX.

The general remark in this case is that, as the number of sessions increases, both packet loss and latency increase. This occurs, as more sessions result in increased traffic, which is followed by packet collisions causing packet loss.

To reach a conclusion, an additive approach of weighting factors $(0.8,0.2)$ performs quite well in all cases. 

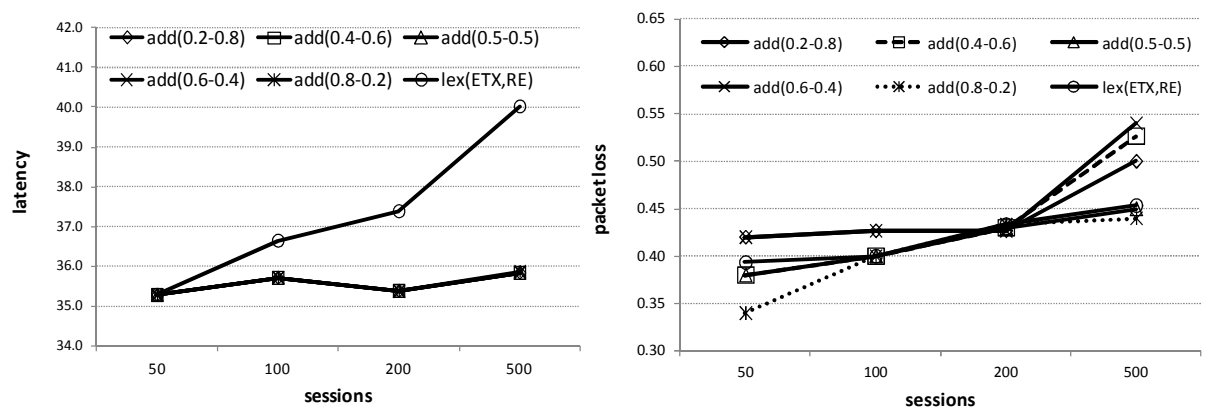

Fig. 5. Latency (left) and packet loss (right) results for additive and lexicographic (ETX, RE) approaches for varying number of session in the presence of 50 misbehaving nodes

\subsection{Security-Related Applications}

Security in public areas as well as disaster management is a critical application area, where the fundamental criterion is the trust-awareness of the deployed nodes, followed by link reliability maximization. For this reason, in this application domain we consider the combination of PFI and ETX as a suitable proposition. Hence, PFI followed by ETX and RE will be used, considering that the network lifetime in these situations is also important.

The results for combinations of PFI, ETX, RE are depicted in Figures 6 and 7 for 50 and 150 misbehaving nodes respectively. (The lex(PFI, ETX, RE) approach leads to high latency and packet loss as already explained in previous sections and for this reason is left out of our investigation.)
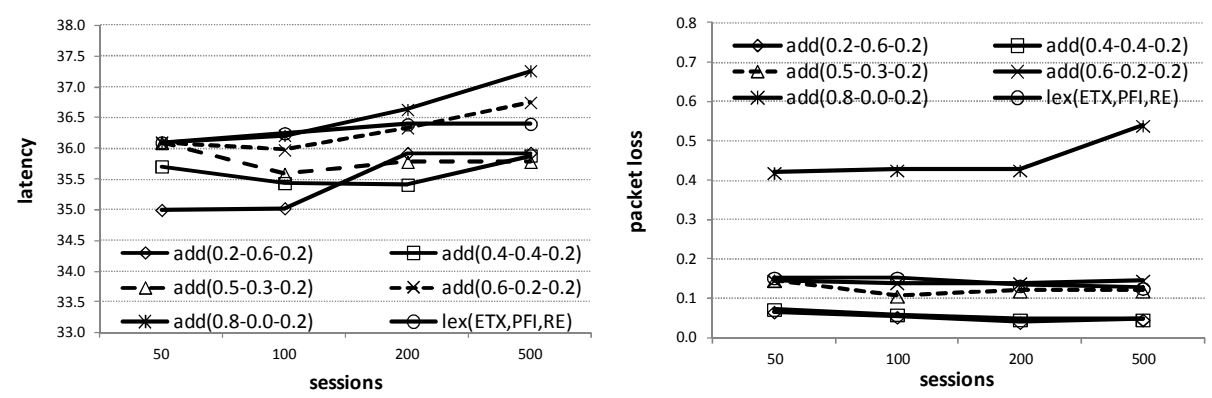

Fig. 6. Latency (left) and packet loss (right) results for additive and lexicographic (PFI, ETX, $\mathrm{RE}$ ) approaches for varying number of session in the presence of 50 misbehaving nodes

The additive composite routing metric with weights equal to $0.8,0$ and 0.2 respectively, (essentially not inspecting PFI) leads to significantly higher packet loss than any other combination, and is considered not acceptable. For all other more "balanced" solutions, the performance differences are not impressive. However, the weight vector $0.4,0.4$ and 0.2 leads to an optimal performance both in terms of latency and packet loss, performing better than the lex(ETX, PFI, RE) approach. 

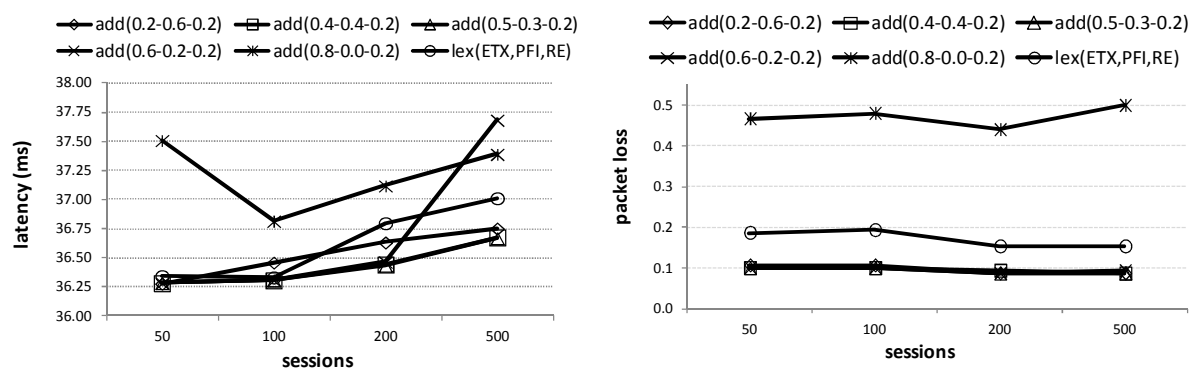

Fig. 7. Latency (left) and packet loss (right) results for additive and lexicographic (PFI, ETX, $\mathrm{RE})$ approaches for varying number of session in the presence of 150 misbehaving nodes

The same qualitative results are observed for 150 misbehaving nodes, as depicted in Figure 7. Again, the additive composition approach with strong weight assigned to ETX and not taking into account PFI (i.e. weight vector equal to $0.8,0,0.2$ ) leads to high latency and packet loss. The best performance for both latency and packet loss is observed for the additive composite metric with weight equal to $0.4,0.4$ and 0.2 respectively.

Comparing Figures 6 and 7, we can conclude that the existence of more misbehaving nodes affects mainly the packet loss than the latency. The latency slightly increases as the misbehaving nodes increase (since the traffic has to travel longer paths to avoid misbehaving nodes), while comparing the best packet loss for 50 and 150 misbehaving nodes, a difference of $5 \%$ has been measured.

\section{Conclusions}

In this paper, we have discussed the implications of current networking trends on Wireless Sensor Networks and more specifically, we have focused on the trend to use the deployed WSN infrastructure to support additional (most probably different) applications than the originally targeted. To efficiently implement the Virtual Sensor Networking paradigm over the resource constrained WSN substrate, Quality of Service handling is mandatory.

Although during the last decade large number of routing protocols and an even larger number of routing metrics have been proposed, these have targeted the satisfaction of network and application-specific QoS and operational requirements. In the framework of the VITRO project, we have designed a RPL-based routing solution which enables the construction of different paths between any pair of nodes to be followed by traffic from different applications to better satisfy the QoS needs of each application. To do so, different routing metrics are adopted per application for the definition of the corresponding path. Furthermore, considering that each application may demand the optimization of more than one performance aspect, we have defined routing metric and metric composition approaches that can serve diverse application requirements while guaranteeing convergence to optimal paths. 
Based on the formally defined routing algebra formalism, presented in [2]-[3] andcomputer simulation results which have been validated through the VITRO testbed, we have shown that:

1. Using different routing metrics, different performance is observed over the same infrastructure and network load scenario. For example, the latency observed considering RSSI routing metric is almost double than the one observed when the hop count is used to decide the routes. For this reason, the application designer should identify the performance aspects that should be optimized in order to support the QoS that better suits the application needs.

2. In case that more than one performance aspects need to be optimized, there are two ways to combine two or more routing metrics: the lexical and the additive approach, where for the additive approach we consider that weight factors can regulate the emphasis placed on each primary routing metric. The additive approach leads to a more "balanced" performance and is thus considered a proper solution for a system designer. The lexical composition approach is suitable in cases when one wishes to optimize two aspects but there is a strict priority of one over the other.

3. For delay sensitive applications, such as the energy consumption control applications, the combination of $\mathrm{HC}$ and RE satisfies the QoS requirements. Namely, either a lexical (HC, RE) combination or an additive (HC, RE) approach, where the $\mathrm{RE}$ metric weighting factor will not exceed 0.4 is the optimal choice for this application domain.

4. For applications sensitive on security threats and delay performance, such as the traffic control applications over a city, the $\mathrm{HC}$ and the trust-related (PFI) metric need to be combined. More specifically, any combination of $\mathrm{HC}$ and PFI in additive manner leads to stable performance and the weighting factors slightly affect the achieved performance.

5. For applications sensitive to link reliability, such as the applications targeting the logistics domain (and product localization in large areas), the combination of ETX and RE is suitable with an additive approach (using weighting factors equal to $(0.8$, 0.2 ) performs quite well in all cases).

6. Finally, for application sensitive to link reliability and security, as is the case where WSNs are used for security in public areas and disaster management, the trustrelated PFI metric has to be combined with ETX (for link reliability) and RE for prolonged network lifetime. In this case, the best performance in terms of both latency and packet loss is observed for the additive composite metric with weight equal to $0.4,0.4$ and 0.2 respectively.

To this end, it is obvious that there is not a "one-fits-all" solution if WSNs are shared between multiple applications with diverse requirements. If the system is lightly loaded and no misbehaving nodes exist, then adopting simply the hop count metric would satisfy all requirements, but this is a rather simplistic approach, contradicting the assumption that the WSN will be shared among multiple applications. On the other hand, combining all the defined routing metrics would result in lack of emphasis 
on specific performance aspects. Prospective users/designers should take into account the above provided guidelines to achieve their goals.

Acknowledgment. The work presented in this paper was partially supported by the EU-funded FP7 211998 VITRO project.

Open Access. This article is distributed under the terms of the Creative Commons Attribution Noncommercial License which permits any noncommercial use, distribution, and reproduction in any medium, provided the original author(s) and source are credited.

\section{References}

1. Sarakis, L., Zahariadis, T., Leligou, H.C., Dohler, M.: A Framework for Service Provisioning in Virtual Sensor Networks. EURASIP Journal on Wireless Communications and Networking (April 2012), doi:10.1186/1687-1499-2012-135

2. Karkazis, P., Trakadas, P., Leligou, H.C., Sarakis, L., Papaefstathiou, I., Zahariadis, T.: Evaluating routing metric composition approaches for QoS differentiation in low power and lossy networks. In: Wireless Networks. Springer, doi:10.1007/s11276-012-0532-2.

3. Velivasaki, T.-H., Karkazis, P., Zahariadis, T., Trakadas, P., Capsalis, C.: Trust-aware and Link-reliable routing metric composition for Wireless sensor networks. Transactions on Emerging Telecommunications Technologies (October 11, 2012), doi:10.1002/ett.2592

4. IETF, RFC6550. RPL: IPv6 Routing Protocol for Low power and Lossy Networks, http://tools.ietf.org/rfc/rfc6550.txt (accessed November 8, 2012)

5. Vasseur, J.P., et al.: IETF, RFC6551. Routing Metrics used for Path Calculation in Low Power and Lossy Networks, http://tools. ietf.org/html/rfc6551 (accessed November 8, 2012)

6. Sobrinho, J.L.: Algebra and algorithms for QoS path computation and hop-by-hop routing in the Internet. IEEE/ACM Trans. Netw. 10(4), 541-550 (2002)

7. Zahariadis, T., Trakadas, P.: Design Guidelines for Routing Metrics Composition in LLN. draft-zahariadis-roll-metrics-composition-04 (November 23, 2012)

8. IETF, RFC 6552, Objective Function Zero for the Routing Protocol for Low-Power and Lossy Networks, RPL (March 2012)

9. IETF, RFC 6719, The Minimum Rank with Hysteresis Objective Function (September 2012)

10. J-Sim official web site (2005), http://code.google.com/p/rpl-jsimplatform/

11. Karkazis, P., Trakadas, P., Zahariadis, T., Hatziefremidis, A., Leligou, H.C.: RPL Modeling in J-Sim Platform. In: 9th International Conference on Networked Sensing Systems (2012) 